\title{
Action and function of Akkermansia muciniphila in microbiome ecology, health and disease
}

\section{Ottman, Noora}

$2017-12$

Ottman , N , Geerlings , S Y , Aalvink , S, de Vos , W M \& Belzer , C 2017 , ' Action and function of Akkermansia muciniphila in microbiome ecology, health and disease ', Best

Practice \& Research: Clinical Gastroenterology , vol. 31 , no. 6 , pp. 637-642 . https://doi.org/10.1016/j.bpg.2017.10

http://hdl.handle.net/10138/301990

https://doi.org/10.1016/j.bpg.2017.10.001

publishedVersion

Downloaded from Helda, University of Helsinki institutional repository.

This is an electronic reprint of the original article.

This reprint may differ from the original in pagination and typographic detail.

Please cite the original version. 


\title{
Action and function of Akkermansia muciniphila in microbiome ecology, health and disease
}

\author{
Noora Ottman ${ }^{\text {b }}$, Sharon Y. Geerlings ${ }^{\text {a }}$, Steven Aalvink ${ }^{\text {a }}$, Willem M. de Vos ${ }^{\text {a, }}$, \\ Clara Belzer ${ }^{\text {a, * }}$ \\ ${ }^{a}$ Wageningen University and Research, Stippeneng 4, 6708WE Wageningen, The Netherlands \\ ${ }^{\mathrm{b}}$ Institute of Environmental Medicine, Karolinska Institutet, 17177 Stockholm, Sweden \\ c Immunobiology Research Program, Department of Bacteriology and Immunology, Haartman Institute, University of Helsinki, Helsinki, Finland
}

\section{A R T I C L E I N F O}

\section{Article history:}

Received 25 August 2017

Received in revised form

22 September 2017

Accepted 10 October 2017

\section{Keywords:}

Akkermansia muciniphila

Microbiome

Mucus

Pharmabiotic

Probiotic

\begin{abstract}
A B S T R A C T
The discovery of Akkermansia muciniphila has opened new avenues for the use of this abundant intestinal symbiont in next generation therapeutic products, as well as targeting microbiota dynamics. A. muciniphila is known to colonize the mucosal layer of the human intestine where it triggers both host metabolic and immune responses. A. muciniphila is particularly effective in increasing mucus thickness and increasing gut barrier function. As a result host metabolic markers ameliorate. The mechanism of host regulation is thought to involve the outer membrane composition, including the type IV pili of A. muciniphila, that directly signal to host immune receptors. At the same time the metabolic activity of A. muciniphila leads to the production of short chain fatty acids that are beneficial to the host and microbiota members. This contributes to host-microbiota and microbe-microbe syntrophy The mucolytic activity and metabolite production make A. muciniphila a key species in the mucus layer, stimulating beneficial mucosal microbial networks.

This well studied member of the microbiota has been studied in three aspects that will be further described in this review: i) A. muciniphila characteristics and mucin adaptation, ii) its role as key species in the mucosal microbiome, and iii) its role in host health.
\end{abstract}

๑) 2017 Published by Elsevier Ltd.

\section{Introduction}

In recent years, it has become evident that the intestinal microbiota can play an essential role in the well being of humans. The composition of microbial communities colonizing the gastrointestinal tract differs according to the prevailing environmental conditions in the gut. Factors such as nutrient supply, transit time, host secretions, and $\mathrm{pH}$ play a role in shaping the gut microbiota [1]. In particular, the mucosa-associated microbiota forms a distinct population in the gut, and is influenced by the close proximity of the epithelial layer and nutrients present in the mucus layer [2-5]. One of the key players in this community is the mucus degrader Akkermansia muciniphila, which has been shown to have important implications on host physiology and microbiome composition [6-8].

The Gram-negative bacterium A. muciniphila was isolated from a

\footnotetext{
* Corresponding author.

E-mail address: clara.belzer@wur.nl (C. Belzer).
}

human fecal sample in a quest to discover new mucin-degrading bacteria [9]. As A. muciniphila is the only intestinal isolate of the deeply rooted Verrucomicrobia phylum, it can be easily detected in meta-omics studies [10]. A. muciniphila represents $1-4 \%$ of the total fecal microbiota starting from early life [11,12], and biopsies show it to be abundantly present in the colonic mucosal layer [13,14]. After its discovery, the levels of $A$. muciniphila in the gut were negatively correlated with numerous diseases, including inflammatory bowel diseases (IBD) [13,15], appendicitis [16], obesity [17,18] and diabetes [19]. Further mechanistic studies have shed more light on the antiinflammatory role of $A$. muciniphila in the gut environment $[6,7,20]$.

Based on these findings, A. muciniphila or its components have been suggested to be a promising candidate for next-generation therapeutic products $[21,22]$. Currently the most convincing evidence of its beneficial effect on health comes from studies linking A. muciniphila to metabolic disorders, such as diabetes and obesity. However, the exact signaling mechanisms by which $A$. muciniphila interacts with the host, and the effect it has on the overall microbial community in the gut, require further investigation. Here, we 
review the latest research concerning: i) A. muciniphila characteristics and mucin adaptation, ii) A. muciniphila role as key species in the mucosal microbiome, and iii) A. muciniphila role in host health (Fig. 1).

\section{A. muciniphila characteristics and mucin adaptation}

To gain a competitive advantage in the gut environment, certain bacteria have evolved to metabolize the complex glycans provided by the mucus layer. A. muciniphila encodes a particularly wide repertoire of mucin-degrading enzymes in its relatively small 2.6$\mathrm{Mb}$ genome [23], and can be described as a mucin specialist. As opposed to for example Bacteroides thetaiotaomicron, a glycan generalist [24]. This notion is backed up by the observation that the mucin-degrading genes are conserved in other A. muciniphila strains and Akkermansia species described thus far [25]. Mucus colonization and degradation by $A$. muciniphila result in the production of metabolites such as short-chain fatty acids (SCFA), which may play a role in metabolic heath or inflammatory status of the host [26]. The relative abundance of $A$. muciniphila in the gut is highly responsive to changes. As such, dietary fiber deprivation, weight loss and anorexia increase its levels in the gut [27-29], while a high fat diet decreases it levels [30].

While A. muciniphila is able to individually metabolize mucinderived sugars, such as fucose and $\mathrm{N}$-acetylglucosamine (GlcNAc) in a basal medium with among others large amounts of tryptone, it grows most efficiently when it has access to the entire mucin molecule [31]. Unlike all other members of the Verrucomicrobia, A. muciniphila cannot synthesize threonine. This is yet another indication of its adaptation to life in the mucosal layer, as threonine is one of the most abundant amino acids present in the protein backbone of mucin [32]. Among the mucin-derived sugars, growth on GlcNAc and N-acetylgalactosamine (GalNAc) leads to the highest yields. However, when $A$. muciniphila is grown on a mixture of GlcNAc, GalNAc, fucose and glucose, a non-mucin sugar, it first consumes the non-amino sugars [31]. This may reflect the ease of transport and efficient energy generation of these simple sugars, while GlcNAc and GalNAc are used as nitrogen sources.

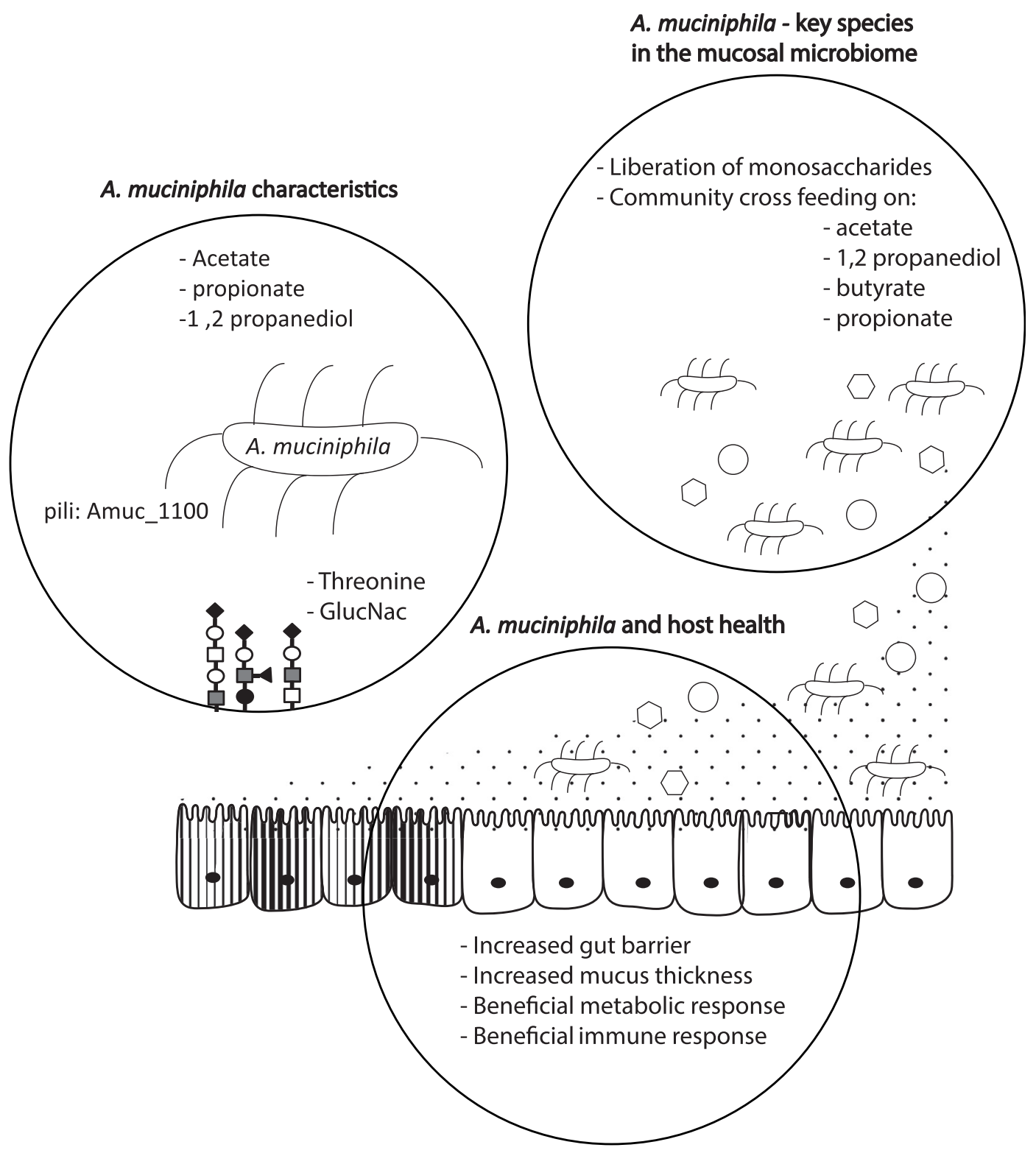

Fig. 1. Three key functions of $A$. muciniphila in the microbiome that could be targets for probiotic and pharmabiotic interventions. 
The availability of mucin in the environment affects A. muciniphila transcriptome and proteome as shown in recent studies [20,27,31]. Numerous genes encoding glycosyl hydrolases and sulfatases that are likely to be involved in mucin degradation were upregulated during $A$. muciniphila growth on mucin as compared to growth on glucose, and a similar response was seen on the proteome level [31]. Likewise, growth on purified mucin $O$ glycans activated $A$. muciniphila genes involved in mucin degradation as compared to growth on GlcNAC, although fewer genes were identified in this case [27]. Environmental variation was also seen in the outer membrane (OM) proteome of $A$. muciniphila, where the abundance of one third of the OM proteins was different between bacterial cells grown on either mucin or glucose [33].

A. muciniphila belongs to the Planctomycetes-VerrucomicrobiaChlamydiae (PVC) superphylum, which contains several members with a compartmentalized cell plan as opposed to a typical Gramnegative membrane architecture [34]. However, a high abundance of proteins involved in OM biogenesis and lipopolysaccharide (LPS) transport were detected in the A. muciniphila OM proteome, supporting structural features commonly present in Gram-negative bacteria [33]. Importantly, analysis of the OM revealed highly abundant proteins predicted to be involved in the formation of the pili-like structures observed in A. muciniphila. Further studies showed that a single protein, termed Amuc_1100 and encoded by a gene cluster also coding for the type 4 pilin secretin Amuc_1098 [33], plays an important role in the capacity of $A$. muciniphila to alleviate metabolic syndrome in mice [7]. These findings highlight the importance of detailed characterization of intestinal bacteria in order to understand the mechanisms behind their reported beneficial effects on the host.

Although A. muciniphila was initially described as strictly anaerobic, it was later discovered to tolerate oxygen [35], and can even benefit from the presence of nanomolar concentrations of oxygen by respiration via a rudimentary electron transport chain [36]. These observations are in line with the fact that $A$. muciniphila colonizes the mucus layer close to the epithelial cells, where low levels of oxygen are present. Oxygen tolerance, along with efficient mucin degradation capacity, demonstrates the adaptation of A. muciniphila to the competitive environment of the mucosal layer. The high efficiency of $A$. muciniphila in foraging host-derived compounds has been demonstrated also in vivo in a study using stable isotope-labeled threonine in a mouse model [37]. The study found that despite the fairly low relative abundance of Akkermansia spp. in the mouse cecum, it was, together with Bacteroides acidifaciens and Mucispirillum shaedleri, the dominant forager of hostderived proteins.

\section{A. muciniphila - key species in the mucosal microbiome}

The mucus layer is a niche in the intestine, which is colonized by specific bacteria. The key species within this mucosal community are able to degrade mucin sugars and the protein backbone [4,5]. The mucus layer consists of a outer gel-forming layer, that provides a habitat for bacteria, and an inner layer, that is devoid of bacteria $[38,39]$. MUC2 is the most abundant gel-forming mucin type in the intestine and it is constructed of a PTS backbone with O-linked glycans [40]. The diverse structure and the different linkages within the glycan chain make it difficult for most micro-organisms to acces the amino acids and mono-saccharides. While for mucin degrading microorganisms it can be used as a source of carbon, energy and nitrogen $[5,9,41,42]$. Certain specialists, like $B$. thetaiotaomicron and A. muciniphila, have specific enzymes to degrade these mucins $[9,13,43,44]$. Especially the sialidases and fucosidases play an important role in mucin degradation, since most of the terminal ends of these oligosaccharide chains are sialic acids or fucose [41]. Sialidases and fucosidases are not commonly encoded in the metagenome of the microbiota. These terminal sugars are thought prevent comprehensive microbial utilization of mucin [45]. The other sugars in the oligosaccharide chain are more easily degraded by members of the microbiota [45].

It should be noted that not all microbes that are found in the mucus layer have the ability degrade mucin. They depend on the mucolytic activity of other microbiota members to colonize the mucus layer [46]. Degradation of the mucin protein backbone and the release of sugars from the glycan chains, provide carbon and nitrogen for the bacteria that do not produce these enzymes $[23,47]$. The butyrate-producers Faecalibacterium prausnitzii, Eubacterium rectale, Roseburia intestinalis and Anaerostipes caccae are examples of species that do not have the ability to degrade mucus, but are commonly found in the mucus layer [8,48-50]. Since butyrate is a source of energy for the colonocytes, the production of butyrate by these organisms nearby the epithelium layer is a useful benefit for the host [51-53]. An actual syntrophy between A. muciniphila and various butyrate producers was shown in vitro. The mucolytic activity of $A$. muciniphila lead to accumulation of acetate and mono-saccharides in the medium. These were fermented to butyrate by the cross feeding butyrogens $\mathrm{A}$. caccae, $\mathrm{Eu}$ bacterium hallii and $F$. prausnizii [8]. Furthermore, when A. muciniphila and E. hallii were co-cultured there was a mutualistic relationship to the benefit of both partners. E. hallii produced pseudovitamin B12 which stimulated the methylmalonyl pathway and hence propionate production of $A$. muciniphila [8]. At the same time, A. muciniphila also produced 1,2 propanediol from fucose which was metabolized by $E$. hallii to propionate $[8,54,55]$. Both of these conversions resulted in the production of propionate, which plays an important role in host gut homeostasis and satiety [52].

Another functional symbiosis of this mucosal key species might be the sulfate that $A$. muciniphila was found to release from the mucin [56]. A. muciniphila abundantly produces sulfatases during mucin degradation [20]. Sulfate reducing bacteria (SRB) are vast colonizers of the mucosal layer of the colon [57]. An in vitro study showed that SRB's may reduce the sulfate released from mucin [58]. Even though these SRB are positively associated with inflammation [59], the actual correlation between hydrogen sulfide and diseases still needs more research, notably since hydrogen sulfide is also neurotransmitter $[60,61]$. Interestingly, A. muciniphila might be able to use hydrogen sulfide for cysteine production, because the cysteine biosynthesis pathway is predicted in the genome, where it uses hydrogen sulfide for the production of L-cysteine. In this way it could control hydrogen sulfide concentrations and potentially limit the toxicity of SRB in the mucin layer.

The microbial ecology at the mucosal layer is essential in understanding the role of the microbiota in health and disease. A. muciniphila can be considered a key species of the mucosal layer, providing the microbial community with mono- and oligosaccharides, SCFA production, and by detoxifying hydrogen sulfide. How the actual mucosal microbial community reaches its stability and is associated with mucosal health needs further investigation.

\section{A. muciniphila host interaction and health}

The presence of $A$. muciniphila in the gut was found to be correlated to a healthy intestine and inversely correlated to many states of disease [13,15-19]. The studies that report a negative correlation of $A$. muciniphila abundance with disease include intestinal disorders, such IBD, but also other diseases, such as autism, 
atopy, and obesity and related diseases. The majority of studies however, have addressed a beneficial role of Akkermansia spp in the prevention and amelioration of metabolic syndrome. As such, A. muciniphila is one of the few microbiota members that is shown to have an actual causal relationship with improved host health in mouse models [7]. Unfortunately, the culturing and preserving techniques for A. muciniphila are not yet sufficient for it to be used as a supplement. However, current $A$. muciniphila research also focuses on the preparation and preservation process of viable and pasteurized cells and cell products for their use in therapeutic interventions [7,62,63].

In terms of microbiota as a potential therapeutic target, the latest data suggest that $A$. muciniphila could potentially be used in treatment for type 2 diabetes (T2D) [64]. In a first study, a decrease of $A$. muciniphila was observed in leptin-deficient mice compared to lean mice $[6,30]$. In addition, a decrease of $A$. muciniphila, to a lesser extent than in obese mice, was also found in mice supplemented with a high-fat diet (HFD) that are obese and develop T2D-like symptoms $[6,65,66]$. The administration of A. muciniphila to HFDfed mice without administration of metformin, which lowers the glucose levels in blood, resulted in a significantly enhanced glucose tolerance and weakened adipose tissue inflammation. Treatment of mice with either live or pasteurized A. muciniphila cells further strengthens the evidence of the effect of this microorganism on metabolic and immune health [7]. Ex vivo experiments using mouse gut organoids showed that $A$. muciniphila metabolites specifically affect various transcription factors and genes involved in cellular lipid metabolism and satiety [67]. Based on these results, increasing Akkermansia levels in the gut could influence anti-diabetic effects of the anti-diabetic drug metformin. In fact metformin was found to increase the levels of Akkermansia spp populations in mice both on a HFD and normal diet $[64,68]$. In humans Akkermansia spp was found to be more abundant in T2D patients compared to healthy controls [69]. However, in newly diagnosed T2D and pre-diabetes patients, a lower abundance of Akkermansia spp was observed [19]. The differential results in these studies could be due to metformin, which can stimulate A. muciniphila [64,68]. Preliminary results of a human clinical trial treating obese individuals with A. muciniphila indicated this organism can be safely used in humans [7]. Altogether, these studies indicate a health-promoting role of A. muciniphila, leading to its possible use as a therapeutic microbe.

The abundance of $A$. muciniphila was also found to be negatively correlated to dietary fat intake [70]. Treatment of mice with A. muciniphila normalized diet-induced metabolic endotoxaemia, adiposity and the adipose tissue markers [6]. Furthermore, the A. muciniphila treatment resulted in reduced body weight gain and body composition even though the food intake did not change [6]. In humans an improved metabolic profile was observed for individuals harboring more A. muciniphila at baseline [18]. In addition, a negative association of $A$. muciniphila with serum total and LDL cholesterol was found in obese Danish women. The reduction in gluconeogenesis and insulin resistance index suggests a key role of A. muciniphila in metabolic inflammation, barrier function and fat storage [6].

A compromised gut barrier function is associated with diseases such as IBD and metabolic syndrome. The majority of human studies observed A. muciniphila depletion in IBD mucosa and fecal samples of patients suffering from ulcerative colitis $[13,71]$. The abundance of $A$. muciniphila was found to be positively correlated to the level of mucins in cecum of rodents [72]. A. muciniphila is able to degrade mucins, but was also found to stimulate mucin production, mucus thickness, and gut barrier [6,64]. In addition, it was also suggested that the restoration of the gut barrier by
A. muciniphila debilitated atherosclerotic lesions and alleviated inflammation induced by metabolic endotoxaemia [73]. Overall, treatment of mice with $A$. muciniphila resulted in an improved gut barrier function $[6,62,71]$.

A. muciniphila is also described to regulate the immune system which leads to production of antimicrobial peptides and improved gut homeostasis. Administration of A. muciniphila resulted in an enhanced production of antimicrobial peptide Reg $3 \gamma$ in the colon and to lesser extent the ileum [6,74]. In addition, A. muciniphila was found to stimulate the proliferation of anti-inflammatory Treg cells in mice [64]. In an in vitro colonic cell line, A. muciniphila adhered to the epithelium and strengthened the intestinal barrier, where a low pro-inflammatory response was induced in comparison to E. coli strain K12 [35]. IgA has a function in elimination of pathogens and maintaining homeostasis [75]. Inoculation of A. muciniphila together with Clostridium scindens in mice prevented lethality and resulted in reduced detachment of epithelial cells in mice colonized with a variety of bacteria targeted by IgA [76]. Host regulation by A. muciniphila was shown to at least partially depend on its outer membrane proteins that includes abundant proteins involved in transport/secretion and proteins with a role in the formation of pililike structures [20]. The highly abundant outer membrane pili-like protein (Amuc_1100) of A. muciniphila type strain MucT was identified [20]. Amuc_1100 is directly involved in immune regulation and enhances trans-epithelial resistance. These studies indicate an active communication between $A$. muciniphila and the host immune system.

Altogether, these studies suggest the usefulness of A. muciniphila as therapeutic treatment for intestinal disorders by the capability to regulate host metabolic response and to increase gut barrier function.

\section{Summary and conclusions}

The microbiome has become an important target for modulating host health. A. muciniphila is one of the few microbiota members proven to have a direct and beneficial effect on host response (Fig. 1). This microorganism is shown to be effective in immune and metabolic regulation, combined with increase gut barrier function. These effects will lead to overall increased gut and metabolic health of the host. The mechanism underlying the host regulatory effects of $A$. muciniphila are described to be through direct signaling of $\mathrm{OM}$ proteins and production of metabolites, as well as through stimulation of a beneficial microbial community (Fig. 1). The core functions of A. muciniphila physiology due to mucin degradation are production of acetate, propionate, and 1,2-propanediol. Mucin degradation by $A$. muciniphila attracts microbiota members that stimulate host health by for example the production of butyrate and prevention of pathogen colonization. There are clear advances in research focused on A. muciniphila in comparison to other intestinal isolates, because a defined medium and optimized culture conditions have been developed to produce this organism for intervention studies. On top of this the organism is reported to be safe for the use in human trials and the first clinical trial has even been conducted [7]. While various genetic improvements of $A$. muciniphila may be considered, the present regulations on genetically modified micro-organisms do not allow easy market introduction of such improved bacteria in countries of the European Union. Future research should focus on the mechanism on how A. muciniphila effectively modulates the microbiome and host response to find the right way of use for this organism in therapeutic applications. 


\section{Practice points}

- A. muciniphila cannot grow without mucin-derived aminosugars and threonine

- A. muciniphila uses the low oxygen levels at the mucosa for respiratory growth

- Mucosal colonization by A. muciniphila enables association with other beneficial microbes such a various butyrogens

- A decrease in abundance of $A$. muciniphila is found during metabolic syndrome in both preclinical and clinical studies

- Intervention studies with $A$. muciniphila have reported beneficial impact on host metabolism

- Living as well as pasteurized cells and the pili protein Amuc_1100 of $A$. muciniphila are involved in host metabolic regulation

\section{Research agenda}

- Future possibilities of $A$. muciniphila manipulation should make it a model organism for Verrucomicrobia in the microbiome

- The role of $A$. muciniphila in microbiome ecology should be further investigated

- Further research should focus on the metabolic regulation in humans

- More insight is needed into the underlying mechanisms of $A$. muciniphila host metabolic regulation

\section{Conflict of interest}

No relevant conflict of interest has been declared by the authors.

\section{Acknowledgement}

This work was supported by Netherlands Organisation for Scientific Research (Spinoza Award and SIAM Gravity Grant 024.002.002).

\section{References}

[1] Gerritsen J, Smidt H, Rijkers GT, de Vos WM. Intestinal microbiota in human health and disease: the impact of probiotics. Genes Nutr 2011;6:209-40.

[2] Belkaid Y, Hand TW. Role of the microbiota in immunity and inflammation. Cell 2014;157:121-41.

[3] Duerr CU, Hornef MW. The mammalian intestinal epithelium as integral player in the establishment and maintenance of host-microbial homeostasis. Semin Immunol 2012:24:25-35.

[4] Ouwerkerk JP, de Vos WM, Belzer C. Glycobiome: bacteria and mucus at the epithelial interface. Best Pract Res Clin Gastroenterol 2013;27:25-38.

[5] Koropatkin NM, Cameron EA, Martens EC. How glycan metabolism shapes the human gut microbiota. Nat Rev Microbiol 2012;10:323-35.

[6] Everard A, Belzer C, Geurts L, Ouwerkerk JP, Druart C, Bindels LB, et al. Crosstalk between Akkermansia muciniphila and intestinal epithelium controls diet-induced obesity. Proc Natl Acad Sci U. S. A 2013;110:9066-71.

[7] Plovier H, Everard A, Druart C, Depommier C, Van Hul M, Geurts L, et al. A purified membrane protein from Akkermansia muciniphila or the pasteurized bacterium improves metabolism in obese and diabetic mice. Nat Med 2017;23:107-13.

[8] Belzer C, Chia L, Aalvink S, Chamlagain BVP, de Vos WM. Microbial metabolic networks at the mucus layer lead to diet independent butyrate -and vitamin
B12 production by intestinal symbionts. 2017.

[9] Derrien M, Vaughan EE, Plugge CM, de Vos WM. Akkermansia muciniphila gen. nov., sp. nov., a human intestinal mucin-degrading bacterium. Int J Syst Evol Microbiol 2004;54:1469-76.

[10] Rooijers K, Kolmeder C, Juste C, Dore J, de Been M, Boeren S, et al. An iterative workflow for mining the human intestinal metaproteome. BMC Genomics 2011;12:6.

[11] Collado MC, Derrien M, Isolauri E, de Vos WM, Salminen S. Intestinal integrity and Akkermansia muciniphila, a mucin-degrading member of the intestinal microbiota present in infants, adults, and the elderly. Appl Environ Microbiol 2007;73:7767-70.

[12] Derrien M, Collado MC, Ben-Amor K, Salminen S, de Vos WM. The mucin degrader Akkermansia muciniphila is an abundant resident of the human intestinal tract. Appl Environ Microbiol 2008;74:1646-8.

[13] Png CW, Linden SK, Gilshenan KS, Zoetendal EG, McSweeney CS, Sly LI, et al. Mucolytic bacteria with increased prevalence in IBD mucosa augment in vitro utilization of mucin by other bacteria. Am J Gastroenterol 2010;105:2420-8.

[14] Lyra A, Forssten S, Rolny P, Wettergren Y, Lahtinen SJ, Salli K, et al. Comparison of bacterial quantities in left and right colon biopsies and faeces. World J Gastroenterol 2012;18:4404-11.

[15] Rajilic-Stojanovic M, Shanahan F, Guarner F, de Vos WM. Phylogenetic analysis of dysbiosis in ulcerative colitis during remission. Inflamm Bowel Dis 2013;19:481-8.

[16] Swidsinski A, Dorffel Y, Loening-Baucke V, Theissig F, Ruckert JC, Ismail M, et al. Acute appendicitis is characterised by local invasion with Fusobacterium nucleatum/necrophorum. Gut 2011;60:34-40.

[17] Karlsson CL, Onnerfalt J, Xu J, Molin G, Ahrne S, Thorngren-Jerneck K. The microbiota of the gut in preschool children with normal and excessive body weight. Obes (Silver Spring) 2012;20:2257-61.

[18] Dao MC, Everard A, Aron-Wisnewsky J, Sokolovska N, Prifti E, Verger EO et al Akkermansia muciniphila and improved metabolic health during a dietary intervention in obesity: relationship with gut microbiome richness and ecology. Gut 2016;65:426-36.

[19] Zhang X, Shen D, Fang Z, Jie Z, Qiu X, Zhang C, et al. Human gut microbiota changes reveal the progression of glucose intolerance. PLoS One 2013;8: e71108.

[20] Ottman N, Reunanen J, Meijerink M, Pietila TE, Kainulainen V, Klievink J, et al. Pili-like proteins of Akkermansia muciniphila modulate host immune responses and gut barrier function. PLoS One 2017;12:e0173004.

[21] Neef A, Sanz Y. Future for probiotic science in functional food and dietary supplement development. Curr Opin Clin Nutr Metab Care 2013;16:679-87.

[22] O'Toole PW, Marchesi JR, Hill C. Next-generation probiotics: the spectrum from probiotics to live biotherapeutics. Nat Microbiol 2017;2:17057.

[23] van Passel MW, Kant R, Zoetendal EG, Plugge CM, Derrien M, Malfatti SA, et al. The genome of Akkermansia muciniphila, a dedicated intestinal mucin degrader, and its use in exploring intestinal metagenomes. PLoS One 2011;6: e16876.

[24] Rogers TE, Pudlo NA, Koropatkin NM, Bell JS, Moya Balasch M, Jasker K, et al. Dynamic responses of Bacteroides thetaiotaomicron during growth on glycan mixtures. Mol Microbiol 2013;88:876-90.

[25] Ouwerkerk JP. Akkermansia species - phylogeny, physiology and comparative genomics. 2016

[26] Puertollano E, Kolida S, Yaqoob P. Biological significance of short-chain fatty acid metabolism by the intestinal microbiome. Curr Opin Clin Nutr Metab Care 2014:17:139-44.

[27] Desai MS, Seekatz AM, Koropatkin NM, Kamada N, Hickey CA, Wolter M, et al. A dietary fiber-deprived gut microbiota degrades the colonic mucus barrier and enhances pathogen susceptibility. Cell 2016;167:1339-53. e1321.

[28] Mack I, Cuntz U, Gramer C, Niedermaier S, Pohl C, Schwiertz A, et al. Weight gain in anorexia nervosa does not ameliorate the faecal microbiota, branched chain fatty acid profiles, and gastrointestinal complaints. Sci Rep 2016;6: 26752.

[29] Louis S, Tappu RM, Damms-Machado A, Huson DH, Bischoff SC. Characterization of the gut microbial community of obese patients following a weightloss intervention using whole metagenome shotgun sequencing. PLoS One 2016;11:e0149564.

[30] Everard A, Lazarevic V, Derrien M, Girard M, Muccioli GG, Neyrinck AM, et al. Responses of gut microbiota and glucose and lipid metabolism to prebiotics in genetic obese and diet-induced leptin-resistant mice. Diabetes 2011;60: 2775-86.

[31] Ottman N, Davids M, Suarez-Diez M, Boeren S, Schaap PJ, Martins dos Santos VAP, et al. Genome-scale model and omics analysis of metabolic capacities of Akkermansia muciniphila reveal a preferential mucin-degrading lifestyle. Appl Environ Microbiol 2017;83(18). https://doi.org/10.1128/ AEM.01014-17.

[32] Schrager J. The chemical composition and function of gastrointestinal mucus. Gut 1970;11:450-6.

[33] Ottman N, Huuskonen L, Reunanen J, Boeren S, Klievink J, Smidt H, et al. Characterization of outer membrane proteome of Akkermansia muciniphila reveals sets of novel proteins exposed to the human intestine. Front Microbiol 2016;7:1157

[34] Lee KC, Webb RI, Janssen PH, Sangwan P, Romeo T, Staley JT, et al. Phylum Verrucomicrobia representatives share a compartmentalized cell plan with members of bacterial phylum Planctomycetes. BMC Microbiol 2009;9:5.

[35] Reunanen J, Kainulainen V, Huuskonen L, Ottman N, Belzer C, Huhtinen H, 
et al. Akkermansia muciniphila adheres to enterocytes and strengthens the integrity of the epithelial cell layer. Appl Environ Microbiol 2015;81:3655-62.

[36] Ouwerkerk JP, van der Ark KC, Davids M, Claassens NJ, Robert Finestra T, de Vos WM, et al. Adaptation of Akkermansia muciniphila to the oxic-anoxic interface of the mucus layer. Appl Environ Microbiol 2016. https://doi.org/ 10.1128/AEM.01641-16.

[37] Berry D, Stecher B, Schintlmeister A, Reichert J, Brugiroux S, Wild B, et al. Host-compound foraging by intestinal microbiota revealed by single-cell stable isotope probing. Proc Natl Acad Sci U. S. A 2013;110:4720-5.

[38] Johansson ME, Larsson JM, Hansson GC. The two mucus layers of colon are organized by the MUC2 mucin, whereas the outer layer is a legislator of hostmicrobial interactions. Proc Natl Acad Sci U. S. A 2011:108(Suppl 1):4659-65.

[39] Johansson ME, Phillipson M, Petersson J, Velcich A, Holm L, Hansson GC. The inner of the two Muc2 mucin-dependent mucus layers in colon is devoid of bacteria. Proc Natl Acad Sci U. S. A 2008;105:15064-9.

[40] Moran AP, Gupta A, Joshi L. Sweet-talk: role of host glycosylation in bacterial pathogenesis of the gastrointestinal tract. Gut 2011;60:1412-25.

[41] Tailford LE, Crost EH, Kavanaugh D, Juge N. Mucin glycan foraging in the human gut microbiome. Front Genet 2015;6:81.

[42] Derrien M, van Passel MW, van de Bovenkamp JH, Schipper RG, de Vos WM, Dekker J. Mucin-bacterial interactions in the human oral cavity and digestive tract. Gut Microbes 2010;1:254-68.

[43] Backhed F, Ley RE, Sonnenburg JL, Peterson DA, Gordon JI. Host-bacterial mutualism in the human intestine. Science 2005;307:1915-20.

[44] Xu J, Bjursell MK, Himrod J, Deng S, Carmichael LK, Chiang HC, et al. A genomic view of the human-Bacteroides thetaiotaomicron symbiosis. Science 2003;299:2074-6.

[45] Ng KM, Ferreyra JA, Higginbottom SK, Lynch JB, Kashyap PC, Gopinath S, et al. Microbiota-liberated host sugars facilitate post-antibiotic expansion of enteric pathogens. Nature 2013:502:96-9.

[46] Hooper LV, Macpherson AJ. Immune adaptations that maintain homeostasis with the intestinal microbiota. Nat Rev Immunol 2010;10:159-69.

[47] Robinson LS, Lewis WG, Lewis AL. The sialate O-acetylesterase EstA from gut Bacteroidetes species enables sialidase-mediated cross-species foraging of 9O-acetylated sialoglycans. J Biol Chem 2017. https://doi.org/10.1074/ jbc.M116.769232.

[48] Van den Abbeele P, Belzer C, Goossens M, Kleerebezem M, De Vos WM, Thas O, et al. Butyrate-producing Clostridium cluster XIVa species specifically colonize mucins in an in vitro gut model. ISME J 2013;7:949-61.

[49] Lopez-Siles M, Khan TM, Duncan SH, Harmsen HJ, Garcia-Gil LJ, Flint HJ. Cultured representatives of two major phylogroups of human colonic Faecalibacterium prausnitzii can utilize pectin, uronic acids, and host-derived substrates for growth. Appl Environ Microbiol 2012;78:420-8.

[50] Chen L, Wang W, Zhou R, Ng SC, Li J, Huang M, et al. Characteristics of fecal and mucosa-associated microbiota in Chinese patients with inflammatory bowel disease, vol. 93; 2014e51. Medicine (Baltimore).

[51] Thibault R, Blachier F, Darcy-Vrillon B, de Coppet P, Bourreille A, Segain JP. Butyrate utilization by the colonic mucosa in inflammatory bowel diseases: a transport deficiency. Inflamm Bowel Dis 2010;16:684-95.

[52] Koh A, De Vadder F, Kovatcheva-Datchary P, Backhed F. From dietary fiber to host physiology: short-chain fatty acids as key bacterial metabolites. Cell 2016;165:1332-45.

[53] Gaudier E, Jarry A, Blottiere HM, de Coppet P, Buisine MP, Aubert JP, et al. Butyrate specifically modulates MUC gene expression in intestinal epithelial goblet cells deprived of glucose. Am J Physiol Gastrointest Liver Physiol 2004;287:G1168-74.

[54] Ottman N, Davids M, Suarez-Diez M, Boeren S, Schaap PJ, Martins Dos Santos VAP, et al. Genome-scale model and omics analysis of metabolic capacities of Akkermansia muciniphila reveal a preferential mucin-degrading lifestyle. Appl Environ Microbiol 2017. https://doi.org/10.1128/AEM.0101401017.

[55] Engels C, Ruscheweyh HJ, Beerenwinkel N, Lacroix C, Schwab C. The common gut microbe Eubacterium hallii also contributes to intestinal propionate formation. Front Microbiol 2016;7.
[56] Derrien M. Mucin utilisation and host interactions of the novel intestina microbe Akkermansia muciniphila ( $\mathrm{Ph} \mathrm{D}$ ). Wageningen: Wageningen Universiteit; 2007.

[57] Nava GM, Carbonero F, Croix JA, Greenberg E, Gaskins HR. Abundance and diversity of mucosa-associated hydrogenotrophic microbes in the healthy human colon. ISME J 2012;6:57-70.

[58] Willis CL, Cummings JH, Neale G, Gibson GR. In vitro effects of mucin fermentation on the growth of human colonic sulphate-reducing bacteria. Anaerobe 1996:2:117-22.

[59] Loubinoux J, Bronowicki JP, Pereira IAC, Mougenel JL, Le Faou AE. Sulfatereducing bacteria in human feces and their association with inflammatory bowel diseases. Fems Microbiol Ecol 2002;40:107-12.

[60] Ijssennagger N, van der Meer R, van Mil SWC. Sulfide as a mucus barrierbreaker in inflammatory bowel disease? Trends Mol Med 2016;22:190-9.

[61] Kimura H. Signaling of hydrogen sulfide and polysulfides. Antioxid Redox Signal 2015;22:347-9.

[62] Ouwerkerk JP, Aalvink S, Belzer C, De Vos WM. Preparation and preservation of viable Akkermansia muciniphila cells for therapeutic interventions. Benef Microbes 2017;8:163-9.

[63] van der Ark KCH, van Heck RGA, Martins Dos Santos VAP, Belzer C, de Vos WM. More than just a gut feeling: constraint-based genome-scale metabolic models for predicting functions of human intestinal microbes. Microbiome 2017:5:78.

[64] Shin NR, Lee JC, Lee HY, Kim MS, Whon TW, Lee MS, et al. An increase in the Akkermansia spp. population induced by metformin treatment improves glucose homeostasis in diet-induced obese mice. Gut 2014;63:727-35.

[65] Nobel YR, Cox LM, Kirigin FF, Bokulich NA, Yamanishi S, Teitler I, et al. Metabolic and metagenomic outcomes from early-life pulsed antibiotic treatment. Nat Commun 2015;6:7486.

[66] Cox LM, Yamanishi S, Sohn J, Alekseyenko AV, Leung JM, Cho I, et al. Altering the intestinal microbiota during a critical developmental window has lasting metabolic consequences. Cell 2014;158:705-21.

[67] Lukovac S, Belzer C, Pellis L, Keijser BJ, de Vos WM, Montijn RC, et al. Differential modulation by Akkermansia muciniphila and Faecalibacterium prausnitzii of host peripheral lipid metabolism and histone acetylation in mouse gut organoids. MBio 2014;5.

[68] Lee H, Ko G. Effect of metformin on metabolic improvement and gut microbiota. Appl Environ Microbiol 2014;80:5935-43.

[69] Qin J, Li Y, Cai Z, Li S, Zhu J, Zhang F, et al. A metagenome-wide association study of gut microbiota in type 2 diabetes. Nature 2012;490:55-60.

[70] Brahe LK, Le Chatelier E, Prifti E, Pons N, Kennedy S, Hansen T, et al. Specific gut microbiota features and metabolic markers in postmenopausal women with obesity. Nutr Diabetes 2015;5:e159.

[71] Loonen LM, Stolte EH, Jaklofsky MT, Meijerink M, Dekker J, van Baarlen P, et al. REG3gamma-deficient mice have altered mucus distribution and increased mucosal inflammatory responses to the microbiota and enteric pathogens in the ileum. Mucosal Immunol 2014;7:939-47.

[72] Van den Abbeele P, Gerard P, Rabot S, Bruneau A, El Aidy S, Derrien M, et al. Arabinoxylans and inulin differentially modulate the mucosal and luminal gut microbiota and mucin-degradation in humanized rats. Environ Microbiol 2011;13:2667-80.

[73] Li J, Lin S, Vanhoutte PM, Woo CW, Xu A. Akkermansia muciniphila protects against atherosclerosis by preventing metabolic endotoxemia-induced inflammation in apoe-/- mice. Circulation 2016;133:2434-46.

[74] Derrien M, Van Baarlen P, Hooiveld G, Norin E, Muller M, de Vos WM. Modulation of mucosal immune response, tolerance, and proliferation in mice colonized by the mucin-degrader Akkermansia muciniphila. Front Microbiol 2011;2:166.

[75] Alexander KL, Targan SR, Elson 3rd CO. Microbiota activation and regulation of innate and adaptive immunity. Immunol Rev 2014;260:206-20.

[76] Kau AL, Planer JD, Liu J, Rao S, Yatsunenko T, Trehan I, et al. Functional characterization of IgA-targeted bacterial taxa from undernourished Malawian children that produce diet-dependent enteropathy. Sci Transl Med 2015;7. 276ra224. 